\title{
Three-Dimensional Stability Characteristics of Finite Electrified Conducting Fluids Streaming through a Porous Medium
}

\author{
T. M. N. Metwaly (i) and Zakaria M. Gharsseldien (iD \\ Department of Mathematics, College of Arts and Science, Prince Sattam Bin Abdulaziz University, \\ Wadi Adwassir 11991 (54), Saudi Arabia \\ Correspondence should be addressed to T. M. N. Metwaly; t.nour@psau.edu.sa
}

Received 9 November 2020; Revised 23 November 2020; Accepted 4 December 2020; Published 15 December 2020

Academic Editor: Velusamy Vijayakumar

Copyright (c) 2020 T. M. N. Metwaly and Zakaria M. Gharsseldien. This is an open access article distributed under the Creative Commons Attribution License, which permits unrestricted use, distribution, and reproduction in any medium, provided the original work is properly cited.

\begin{abstract}
A novel procedure is utilized to investigate the surface waves between two finite conducting fluids streaming through a porous medium in the presence of a horizontal electric field. Normal mode analysis is applied to study two- and three-dimension disturbances cases. The quadratic dispersion equation of complex coefficients representing the system is derived and discussed. It is noted that based on appropriate data selections, the stability criteria do not depend on the medium permeability. It is found that electrical conductivities, viscosities, medium porosity, and surface tension enhance the stability of the system while the dimension and the fluid velocities decrease the stability of the system. Finally, the fluid depths have a dual role (stabilizing as well as destabilizing effects) on the system.
\end{abstract}

\section{Introduction}

The Kelvin-Helmholtz instability (KHI) appears if two superposed fluids streaming with a relative horizontal velocity. This instability is very important in describing more kinds of astrophysical and space phenomena involving sheared stream. As a consequence of its connection to geophysical, astrophysical, and laboratory areas [1], it has been examined by numerous contributors. The linear KHI is treated in Chandrasekhar's monogragh [2]. The impact of streaming is destabilized in the linear access in the KHI problem.

Electrohydrodynamics (EHD) can be treated as the branch of fluid mechanics interested in electrical force effects or as the part of EHD that is concerned with the influence of fluid motion on electric fields. Consequently, it is concerned with (electric fields polarized or free charges) interactions in fluids. The fluids may be very good insulators, slight conductors, or even high conductors. A survey on EHD subject with specific reference to several of the advancements in this area is provided by Melcher [3].

In the surface wave phenomena through dielectric fluids when both surface tension and gravity are essential, the capillary waves and the gravity couple to create one wave distinguished by the two influences. By contrast, the effect of an external electric field during conducting fluid flows has many several characteristics and various valuable engineering and physical applications. When surface charges share in the motion of fluids, the arising waves are mentioned as EHD surface waves. The effect of an electric field on the linear stability of a sharp interface dividing two nonconducting dielectric fluids of unlimited range has been investigated by Melcher and Schwarz [4]. A review of the role of interfacial stresses on EHD has been presented by Melcher and Taylor [5] and later the same characteristics have been reviewed by many authors (see Baygents and Baldessare [6] and Rudraiah et al. [7]).

In the linear EHD stability theory, it is well known that the horizontal electric field has a stabilizing effect while the vertical one always has a destabilizing influence [8-12]. EHD stability problems of the interface between conducting fluids in planar and cylindrical geometries have been as of late examined by many authors [13-18].

Flows through a porous medium have been a topic of high interest for the latest many decades. This interest was 
driven by many engineering and geophysical applications in various majors [19]. Many recent works on this topic are investigated by many authors [20-23]. In most earlier investigations on porous media, many researchers considered the treatments based on Darcy's law and Forschheimerextended Darcy's law models. Darcy's law is an experimental formula relating the bulk viscous resistance, the gravitational force, and the pressure gradient in a porous medium.

The KHI for flow in porous media has received great attention in the scientific literature. Sharma and Spanos [24] investigated the instability of the plane interface separating two uniform superposed fluids motion through porous media. El-Sayed [25] analyzed the instability of two superposed viscous streaming fluids through porous media in the presence of a horizontal electric field. For recent surveys regarding the developments of linear and nonlinear electromagnetic flows in porous media, see refs. [26-36].

The goal of the present paper is to investigate the linear stability of two electrically conducting fluids of finite thickness moving through a porous medium under a uniform horizontal electric field. In addition, the uniform flow is taken through three dimensions to be more general and then using the normal modes analysis to obtain the solutions of system parameters. This problem, as far as we know, has not been studied yet. The stability results are presented in figures. All these flows are new and are presented for the first time in the literature.

\section{Problem Description}

We consider the three-dimensional finite amplitude surface waves propagating at the interface $z=0$ that represent the balance situation. The perturbed interface $z=\zeta(x, y, t)$ separates two conducting incompressible fluids that have a uniform thickness. Fluid (1) occupies the lower region $-h_{1}<z<\zeta(x, y, t)$ while fluid (2) occupies the upper region $\zeta(x, y, t)<z<h_{2}$. The two fluids are streaming with uniform velocities $U_{1}$ and $U_{2}$ along the $x$-axis through a porous medium. The system is influenced by a constant horizontal electric field $E_{0}$ in the $x$-direction. The gravitational acceleration $g$ directed normal to the interface. The surface tension forces $T$ are taken into account between the two fluids. We shall denote $\rho_{j}, \mu_{j}, \varepsilon_{j}, \alpha_{j}, E_{j}, \lambda_{1}$, and $m$, $(j=1,2)$ to the fluids densities, viscosities coefficients, dielectric constants, electrical conductivities, electric field elements, permeability, and porosity of the medium. A sketch of the physical problem is given in Figure 1. The disturbed interface may be written as

$$
F(x, y, z, t)=z-\zeta(x, y, t)=0 .
$$

The unit outer perpendicular to the interface is

$$
\mathbf{n}=\frac{\nabla F}{|\nabla F|}=\left[1+\zeta_{x}^{2}+\zeta_{y}^{2}\right]^{-(1 / 2)}\left(-\zeta_{x},-\zeta_{y}, 1\right)
$$

The equation of motion for an incompressible viscous fluid in a porous medium is

$$
\frac{\rho}{m}\left[\frac{\partial \mathbf{v}}{\partial t}+\frac{1}{m}(\mathbf{v} \cdot \nabla) \mathbf{v}\right]=-\nabla p-\rho g \mathbf{e}_{z}-\frac{\mu}{\lambda_{1}} \mathbf{v} .
$$

The continuity equation is

$$
\nabla \cdot \mathbf{v}=0
$$

where $p$ and $\mathbf{v}$ are the pressure and the velocity, respectively. Considering irrotational fluid flows, then the velocity potentials is $\Phi_{j}(x, y, z, t)$ such that

$$
\mathbf{v}_{j}=U_{j} \mathbf{e}_{x}+\nabla \Phi_{j}, \quad(j=1,2),
$$

where $\mathbf{e}_{x}$ is the unit vector in the $x$-direction. The potentials $\Phi_{j}$ satisfy the Laplace's equations:

$$
\begin{gathered}
\nabla^{2} \Phi_{1}=0, \quad \text { in }-h_{1}<z<\zeta(x, y, t), \\
\nabla^{2} \Phi_{2}=0, \quad \text { in } \zeta(x, y, t)<z<h_{2} .
\end{gathered}
$$

It is supposed that, in electrodynamics, the quasi-static approximation is valid. Hence, the electrical equations are

$$
\begin{aligned}
\nabla \times \mathbf{E} & =0, \\
\nabla \cdot(\varepsilon \mathbf{E}) & =q .
\end{aligned}
$$

The charge conservation equation is

$$
\nabla \cdot \mathbf{J}+\frac{\partial q}{\partial t}=0
$$

where $\mathbf{J}=\alpha \mathbf{E}+q \mathbf{v}$ is the free current density (stationary current), $\alpha$ is the electrical conductivity, and $q$ is the free charge density. Therefore, the electric field can be represented as the gradient of electrostatic potentials $\Psi_{j}(x, y, z, t)$ :

$$
E_{j}=E_{0} \mathbf{e}_{x}-\nabla \Psi_{j}, \quad(j=1,2) .
$$

From equations (8) and (10), the scalar electric potentials $\Psi_{j}$ to satisfy the Laplace's equations are as follows:

$$
\begin{gathered}
\nabla^{2} \Psi_{1}=0, \quad \text { in }-h_{1}<z<\zeta(x, y, t), \\
\nabla^{2} \Psi_{2}=0, \quad \text { in } \zeta(x, y, t)<z<h_{2} .
\end{gathered}
$$

\section{The Boundary Conditions}

(1) The vanishing of the normal fluid velocities on both the lower and upper boundaries is

$$
\frac{\partial \Phi_{j}}{\partial z}=0, \quad \text { at } z=(-1)^{j} h_{j},(j=1,2) .
$$

(2) The vanishing of the normal components of the electric fields also on these boundaries is

$$
\frac{\partial \Psi_{j}}{\partial z}=0, \quad \text { at } z=(-1)^{j} h_{j},(j=1,2) .
$$




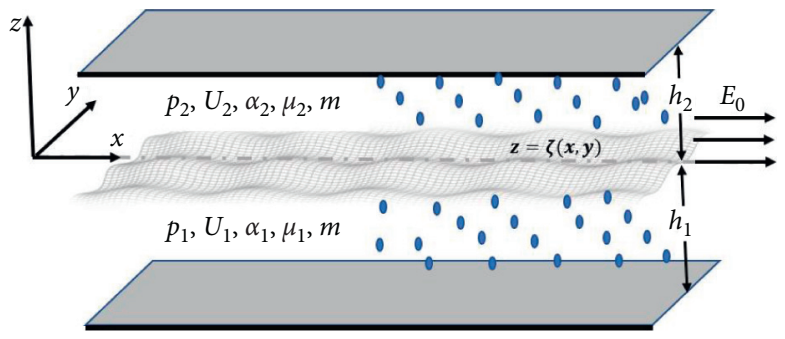

FIGURE 1: A sketch of the physical problem.

(3) The kinematic boundary condition at the interface $z=\zeta(x, y, t)$ is given by

$m \frac{\partial \zeta}{\partial t}-\frac{\partial \Phi_{j}}{\partial z}+\frac{\partial \zeta}{\partial x}\left(U_{j}+\frac{\partial \Phi_{j}}{\partial x}\right)+\frac{\partial \zeta}{\partial y} \frac{\partial \Phi_{j}}{\partial y}=0$

$$
(j=1,2) \text {. }
$$

(4) The tangential electric field component is supposed continuous at the interface $z=\zeta(x, y, t)$ :

$$
\left\|\Psi_{x}\right\|+\zeta_{x}\left\|\Psi_{z}\right\|=0
$$

where $\|f\|=f_{2}-f_{1}$ describes the jump of the quantity $f$ over the interface.

(5) When uniform conductivity of fluids is considered, the problem became more attractive yet difficult. Surface charges play an essential role in such cases; the conduction process in the interface region is a significant factor in many electrical systems. The continuity of stationary current normal to the interface $z=\zeta(x, y, t)$ must lead to charge accumulation on the interface [17]:

$$
\zeta_{x}\left\|\alpha \Psi_{x}\right\|+\zeta_{y}\left\|\alpha \Psi_{y}\right\|-\zeta_{x} E_{0}\|\alpha\|-\left\|\alpha \Psi_{z}\right\|=0 .
$$

(6) The interfacial normal stress component must be continuous at the interface $z=\zeta(x, y, t)$ :

$$
\begin{aligned}
& \frac{1}{m^{2}}\left\|\rho\left(m \Phi_{t}+U \Phi_{x}\right)\right\|+\frac{1}{\lambda_{1}}\|\mu \Phi\| \\
& \quad+\zeta_{x}^{2} E_{0}^{2}\|\varepsilon\|+E_{0}\left\|\varepsilon \Psi_{x}\right\|+2 \zeta_{x} E_{0}\left\|\varepsilon \Psi_{z}\right\| \\
& \quad-\zeta_{x}\left\|\varepsilon \Psi_{x}\right\|-\zeta_{y}\left\|\varepsilon \Psi_{y}\right\|+g \zeta\|\rho\|+T\left(\zeta_{x x}+\zeta_{y y}\right)=0 .
\end{aligned}
$$

\section{Linear Theory and Characteristic Equation}

To study the stability of the problem, first we shall analyze the perturbation quantities into normal modes, and we suppose that all the perturbed quantities take the form

$$
f(r) \exp (i \theta)
$$

where $f(r)$ is any function of $r, \theta=(k x+l y-\omega t)$ is the carrier wave phase, $K=\sqrt{\left(k^{2}+l^{2}\right)}, k$ and $l$ represent the wave number elements in $x$ - and $y$ - directions, respectively, while $\omega, A, i$, and c.c denote the angular frequency, the complex amplitude of the surface wave, the imaginary unit, and the complex conjugate of the previous terms, respectively.

Here, using equation (19) together with equations (6), (7), (11), and (12) with the suitable boundary conditions, the potential function solutions may be written as

$$
\begin{aligned}
\zeta & =A \exp (i \theta)+c \cdot c, \\
\Phi_{1} & =\frac{i\left(k U_{1}-m \omega\right)}{K \sinh K h_{1}} \cosh K\left(z+h_{1}\right) A \exp (i \theta)+c \cdot c, \\
\Phi_{2} & =-\frac{i\left(k U_{2}-m \omega\right)}{K \sinh K h_{2}} \cosh K\left(z-h_{2}\right) A \exp (i \theta)+c \cdot c, \\
\Psi_{1} & =\frac{i k E_{0}\left(\alpha_{2}-\alpha_{1}\right)}{K\left(\alpha_{1} \sigma_{1}+\alpha_{2} \sigma_{2}\right)} \frac{\cosh K\left(z+h_{1}\right)}{\cosh K h_{1}} A \exp (i \theta)+c \cdot c, \\
\Psi_{2} & =\frac{i k E_{0}\left(\alpha_{2}-\alpha_{1}\right)}{K\left(\alpha_{1} \sigma_{1}+\alpha_{2} \sigma_{2}\right)} \frac{\cosh K\left(z-h_{2}\right)}{\cosh K h_{2}} A \exp (i \theta)+c \cdot c .
\end{aligned}
$$

Now, $\sigma_{j}=\tanh K h_{j}(j=1,2)$, and to obtain a nontrivial starting solution, the frequency $\omega$ and the wave number $K$ should satisfy the next characteristic equation:

$$
\begin{aligned}
S(\omega, K)= & \frac{1}{m^{2} K}\left\{\frac{\rho_{1}\left(m \omega-k U_{1}\right)^{2}}{\sigma_{1}}+\frac{\rho_{2}\left(m \omega-k U_{2}\right)^{2}}{\sigma_{2}}\right\} \\
& +\frac{i}{K \lambda_{1}}\left\{\frac{\mu_{1}\left(m \omega-k U_{1}\right)}{\sigma_{1}}+\frac{\mu_{2}\left(m \omega-k U_{2}\right)}{\sigma_{2}}\right\} \\
& -\frac{k^{2} E_{0}^{2}\left(\varepsilon_{2}-\varepsilon_{1}\right)\left(\alpha_{2}-\alpha_{1}\right)}{K\left(\alpha_{1} \sigma_{1}+\alpha_{2} \sigma_{2}\right)}+g\left(\rho_{2}-\rho_{1}\right)-T K^{2}=0 .
\end{aligned}
$$

Equation (21) is simplified in the dispersion relation

$$
a_{0} \omega^{2}+\left(a_{1}+i b_{1}\right) \omega+\left(a_{2}+i b_{2}\right)=0
$$

where 


$$
\begin{aligned}
& a_{0}=\left(\frac{\rho_{1}}{\sigma_{1}}+\frac{\rho_{2}}{\sigma_{2}}\right), \\
& a_{1}=-\frac{2 k}{m}\left(\frac{\rho_{1} U_{1}}{\sigma_{1}}+\frac{\rho_{2} U_{2}}{\sigma_{2}}\right), \\
& b_{1}= \frac{m}{\lambda_{1}}\left(\frac{\mu_{1}}{\sigma_{1}}+\frac{\mu_{2}}{\sigma_{2}}\right), \\
& a_{2}= \frac{k^{2}}{m^{2}}\left(\frac{\rho_{1} U_{1}^{2}}{\sigma_{1}}+\frac{\rho_{2} U_{2}^{2}}{\sigma_{2}}\right)-\frac{k^{2} E_{0}^{2}\left(\varepsilon_{2}-\varepsilon_{1}\right)\left(\alpha_{2}-\alpha_{1}\right)}{\left(\alpha_{1} \sigma_{1}+\alpha_{2} \sigma_{2}\right)} \\
&+g K\left(\rho_{2}-\rho_{1}\right)-T K^{3}, \\
& b_{2}=-\frac{k}{\lambda_{1}}\left(\frac{\mu_{1} U_{1}}{\sigma_{1}}+\frac{\mu_{2} U_{2}}{\sigma_{2}}\right) . \\
& \text { We have the following special cases: }
\end{aligned}
$$

(1) In two semiinfinite nonconducting fluids, equation (22) reduces to the corresponding dispersion relation obtained first by El-Sayed [25]

(2) In two-dimensional nonporous case, equation (22) reduces to the same equation derived by Melcher [8]

(3) In the absence of the electric field and the porosity, equation (22) reduces to the corresponding relation presented by Chandrasekhar [2]

\section{Stability Analysis and Discussion}

Applying here the Routh-Hurwitz stability criterion [37] to equation (22), the system will be linearly stable according to

$$
\begin{aligned}
b_{1} & >0, \\
a_{2} b_{1}^{2}-a_{1} b_{1} b_{2}+a_{0} b_{2}^{2} & \leq 0 .
\end{aligned}
$$

Since $m$ and $\lambda_{1}$ are positive parameters, the first condition in (24) is trivially satisfied. The second inequality is satisfied if

$$
E_{0}^{2} \geq E_{c}^{2}
$$

where

$$
\begin{aligned}
E_{0}^{2}= & \frac{\left(\alpha_{1} \sigma_{1}+\alpha_{2} \sigma_{2}\right)}{k^{2}\left(\varepsilon_{2}-\varepsilon_{1}\right)\left(\alpha_{2}-\alpha_{1}\right)}\left\{\frac{k^{2}\left(U_{2}-U_{1}\right)^{2}\left(\rho_{2} \sigma_{2} \mu_{1}^{2}+\rho_{1} \sigma_{1} \mu_{2}^{2}\right)}{m^{2}\left(\mu_{1} \sigma_{2}+\mu_{2} \sigma_{1}\right)^{2}}\right. \\
& \left.+g K\left(\rho_{2}-\rho_{1}\right)-T K^{3}\right\} .
\end{aligned}
$$

From the previous stability criteria, it is obvious that the permeability $\lambda_{1}$ of the meduim has no effect on the linear stability of the system. The horizontal electric field has a dual role according to the sign of the term $\left(\varepsilon_{2}-\varepsilon_{1}\right)\left(\alpha_{2}-\alpha_{1}\right)$ in contrast with the pure dielectric fluids case, and this was well studied by Moatimid [38].The surface tension has stabilizing effects, while the uniform streaming velocity is strictly destabilizing. Next, we shall make a numerical discussion to clarify the stability of the problem by graphing the transition curves represented by equation (26) in $E_{0}^{2}-k$ plane when $E_{0}^{2}=E_{c}^{2}$. These transition curves that represent the marginal stability state separate the upper stable $S$ regions from the lower $U$ unstable ones. The calculations are made in that case, for a system having $\rho_{1}=0.000365 \mathrm{gr} / \mathrm{cm}^{3}$, $\rho_{2}=0.597 \mathrm{gr} / \mathrm{cm}^{3}, \quad \varepsilon_{1}=1.007 \mathrm{farad} / \mathrm{cm}, \quad \varepsilon_{2}=1.7 \mathrm{farad} / \mathrm{cm}$, $\alpha_{1}=0.2 \mathrm{mho} / \mathrm{cm}, \quad \alpha_{2}=0.6 \mathrm{mho} / \mathrm{cm}, \quad h_{1}=1.5 \mathrm{~cm}$, $h_{2}=3.5 \mathrm{~cm}, \mu_{1}=0.03 \mathrm{~cm}^{2} / \mathrm{sec}, \mu_{2}=0.7 \mathrm{~cm}^{2} / \mathrm{sec}, U_{1}=4 \mathrm{~cm} /$ sec, $U_{2}=20 \mathrm{~cm} / \mathrm{sec}, g=981 \mathrm{~cm} / \mathrm{sec}^{2}, T=0.05 \mathrm{dyn} / \mathrm{cm}$, and $m=0.06 \mathrm{sec} / \mathrm{cm}$. In Figures 2-8, the first dimension expresses disturbance wave number $k$, the second expresses the critical electric field $E_{0}^{2}$, and the third expresses one of the previous parameters.

Figure 2 shows the variation of the electric field $E_{0}^{2}$ versus $k$ for different electrical conductivity values $\alpha_{1}$ and $\alpha_{2}$ in the two-dimension $(l=0)$ and three-dimension $(l=1,2)$ cases. It was clear that, for $l=0$, the stable region increases by increasing $\alpha_{1}$ and $\alpha_{2}$, while it decreases by increasing the dimension $l(l=1,2)$ for small wave number range $(k \leq 6)$ after that the stability does not depend on $l$ since the curves coincide. Also, for any value $l$ and small $E_{0}^{2}$ values, the system is always unstable, while for high $E_{0}^{2}$, the electric field has a stabilizing effect since stable region increases by increasing the electric field $E_{0}^{2}$ and wave number value $k$. Then, both the electrical conductivites $\alpha_{1}$ and $\alpha_{2}$ and the electric field $E_{0}^{2}$ have stabilizing effects, while the dimension $l$ has a destabilizing effect only for small wave number value $k$.

Also the effects of the porosity $m$ of the medium (see Figure 3) as well as the fluid viscosities $\mu_{1}$ and $\mu_{2}$ (see Figure 4) on the stability are found to be similar to the effect of the electrical conductivites $\alpha_{1}$ and $\alpha_{2}$ given in Figure 2 . Thus, each of the porosity $m$ of the medium and the fluid viscosities $\mu_{1}$ and $\mu_{2}$ has stabilizing effects on the system.

In Figure 5, we draw $E_{0}^{2}$ versus $k$ for different surface tension values $T$ in two- and three-dimension disturbances cases. It was clear that, when $l=0$ and for small values of $T$, the stability increases by increasing $T$ while it decreases by increasing the dimension $l$ for small wave numbers $(k \leq 6)$ just like the previous figures. In contrast, for high values of $T, t$ is seen that the stability increases by increasing $T$ and the dimension $l$ for wave numbers range $(k \geqslant 1)$. We conclude that, the dimension $l$ plays a dual role (stablizing and destablizing) when there are large variation in surface tension force between the fluids.

Figure 6 shows the variation of $E_{0}^{2}$ against $k$ for different fluid velocities values $U_{1}$ and $U_{2}$ in two- and three-dimension cases. It is shown that, when $(l=0,1,2)$, the instability increases by increasing the fluid velocities $U_{1}$ and $U_{2}$, and for any fixed values of $U_{1}$ and $U_{2}$, the instability also increases by increasing $l$ for small wave number $(k \leq 6)$ values after which $l$ has no effect on the stability. Therefore, both the fluid velocities $U_{1}$ and $U_{2}$ and the dimension $l$ have destabilizing effects, while for the high wave number value $k$, the dimension $l$ has no effect on the stability.

Finally, Figures 7 and 8 show the effects of the fluid depths $h_{1}$ and $h_{2}$ accompanied with the dimension $l$ on the stability, stabilizing the system for small values of $h_{1}$ and $h_{2}$ (see Figure 7) while destabilizing it for large values of $h_{1}$ and $h_{2}$ (see Figure 8). Thus, the fluid depths and the dimension have a dual role in the system. 


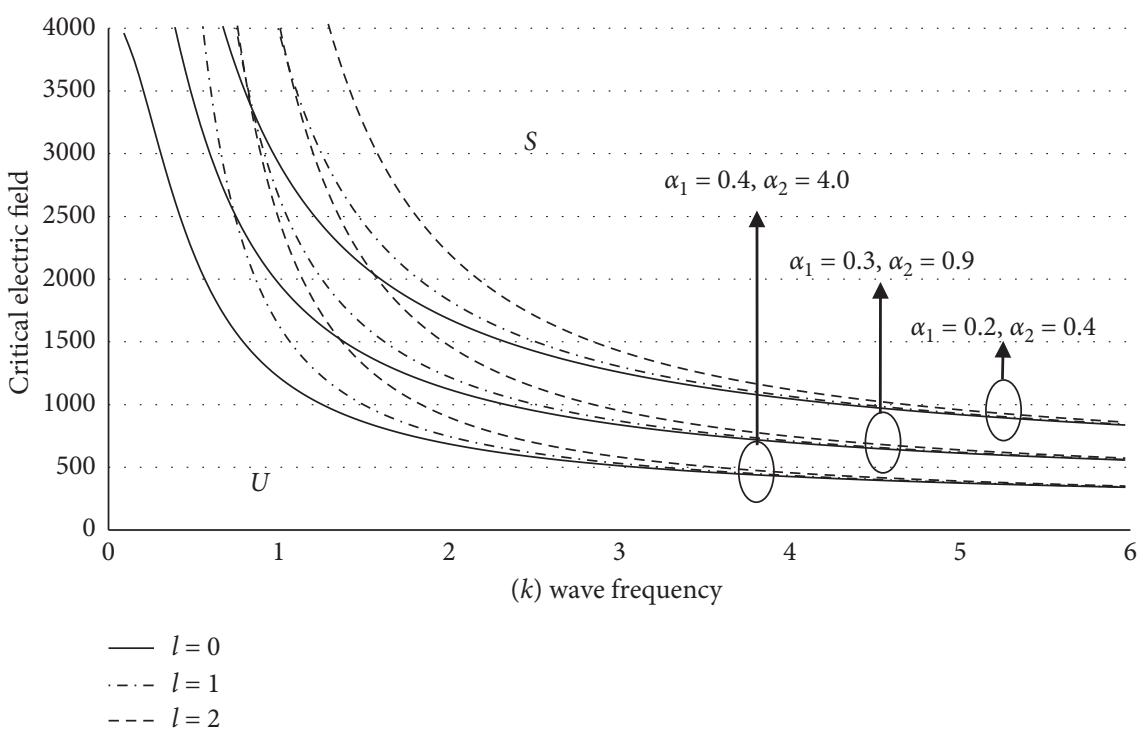

FIgURE 2: Variation of $E_{0}^{2}$ with $k$ for different values of the electrical conductivites $\alpha_{1}$ and $\alpha_{2}$.

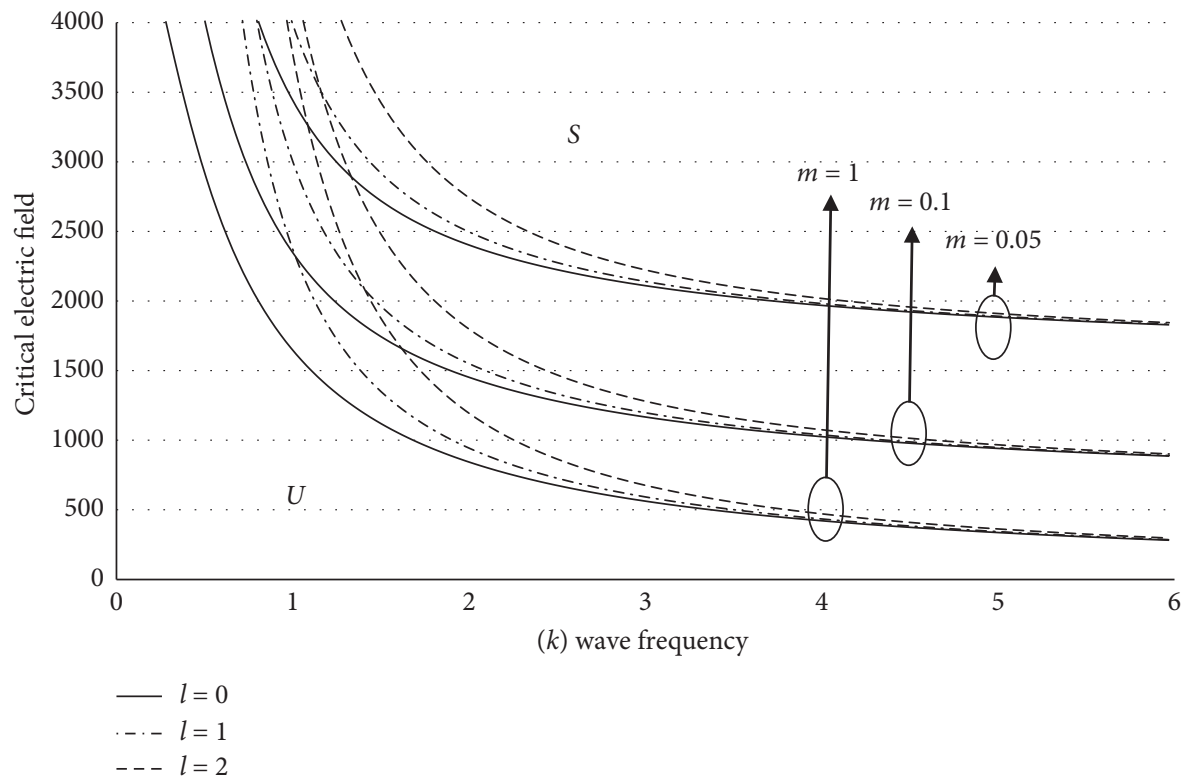

Figure 3: Variation of $E_{0}^{2}$ with $k$ for different values of the porosity of a porous medium $m$. 


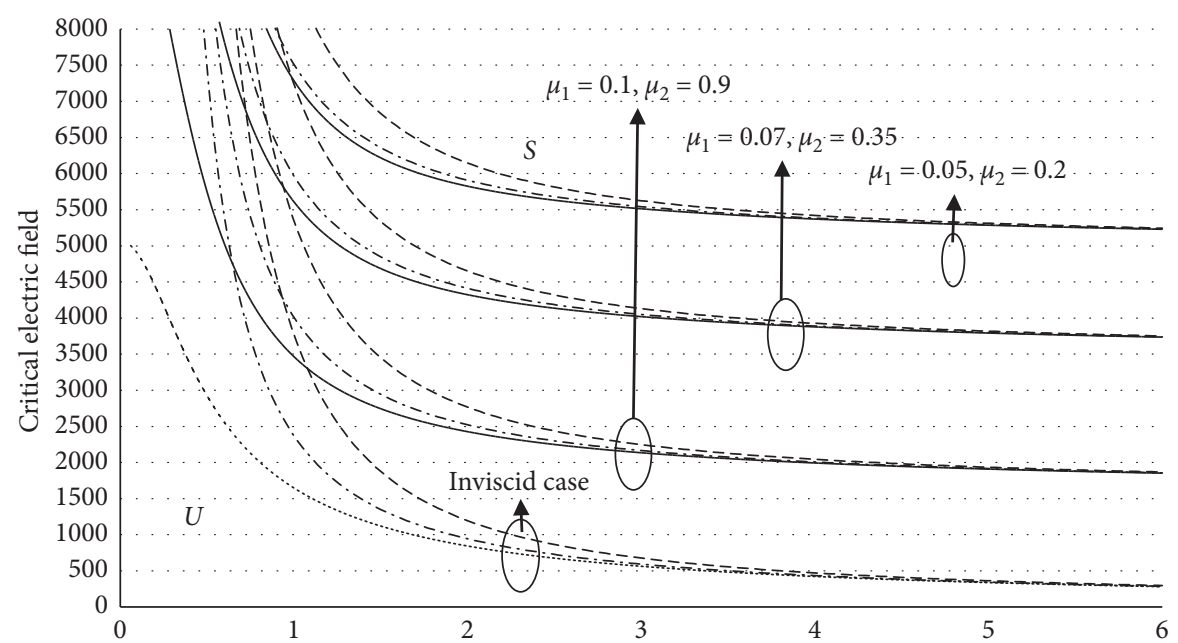

(k) wave frequency

$$
\begin{aligned}
&-l=0 \\
&--l=1 \\
&--l=2
\end{aligned}
$$

FIgURE 4: Variation of with $k$ for different values of the fluid viscosities $\mu_{1}$ and $\mu_{2}$.

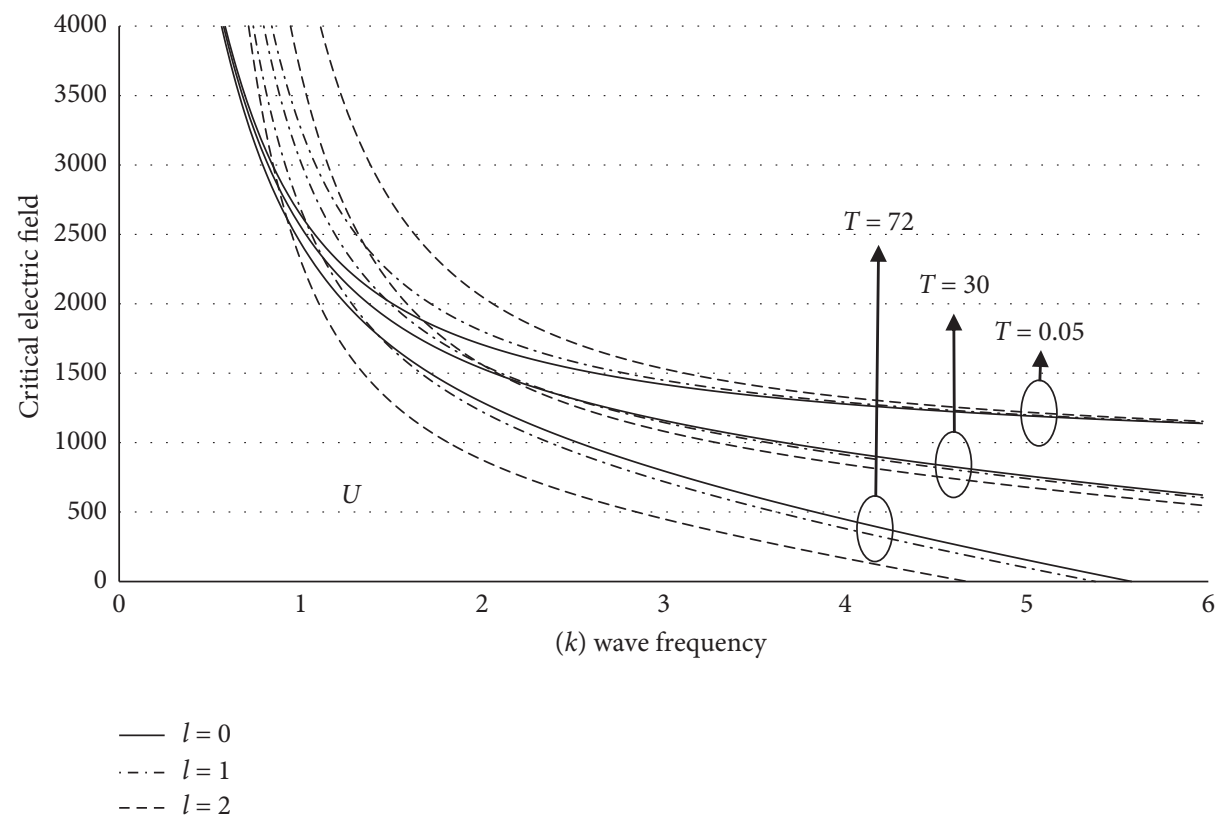

Figure 5: Variation of $E_{0}^{2}$ with $k$ for different values of the surface tension $T$. 


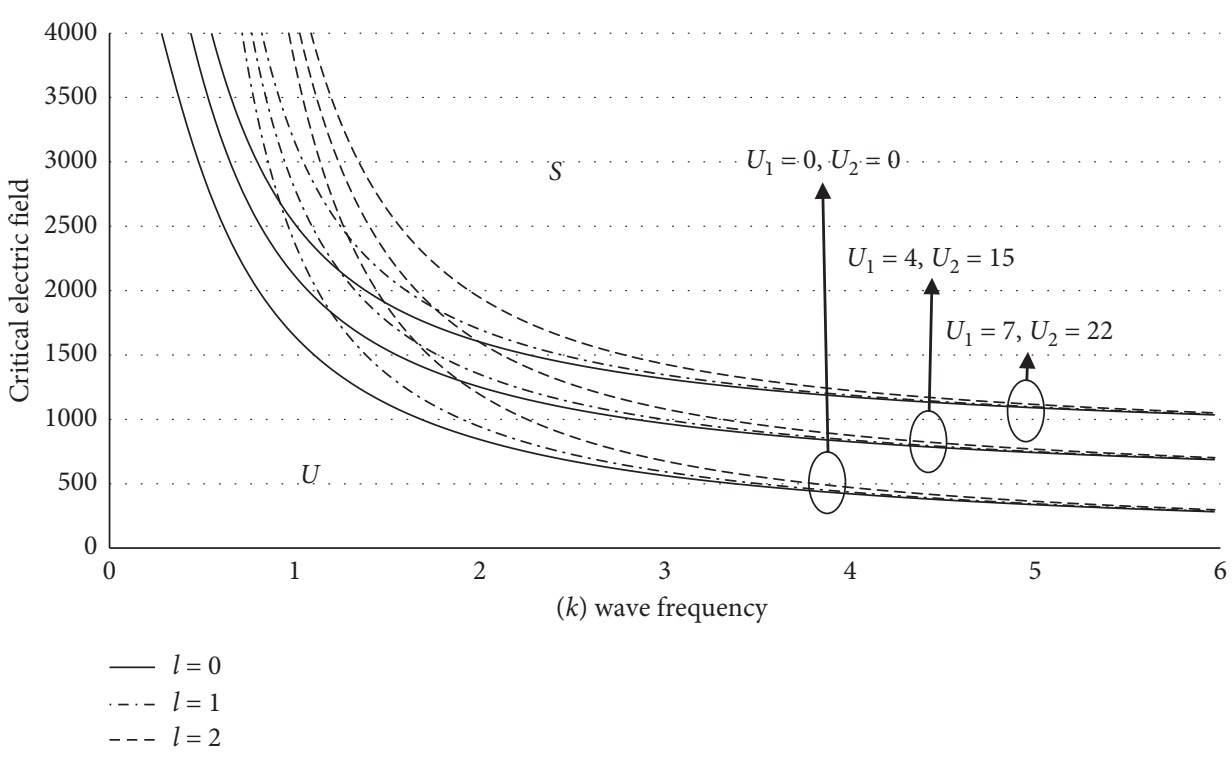

Figure 6: Variation of $E_{0}^{2}$ with $k$ for different values of the fluid velocities $U_{1}$ and $U_{2}$.

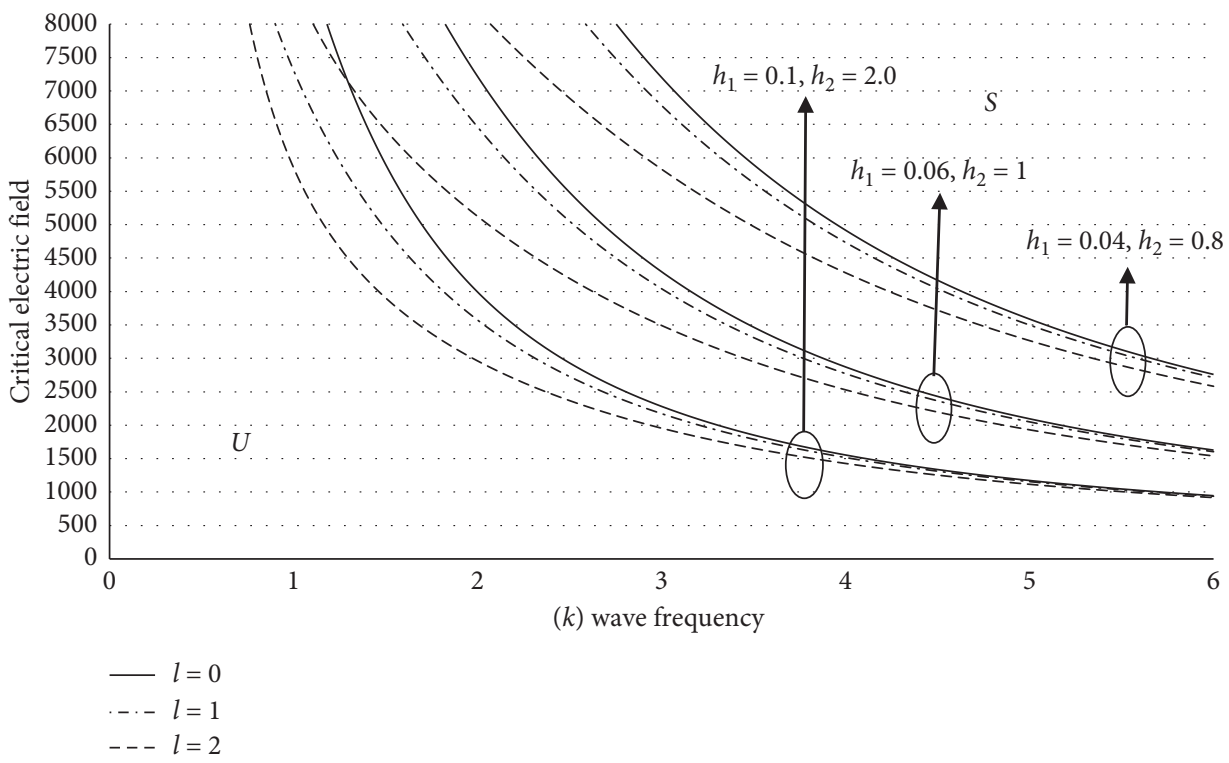

FIGURE 7: Variation of $E_{0}^{2}$ with $k$ for small values of the fluid velocities $h_{1}$ and $h_{2}$. 


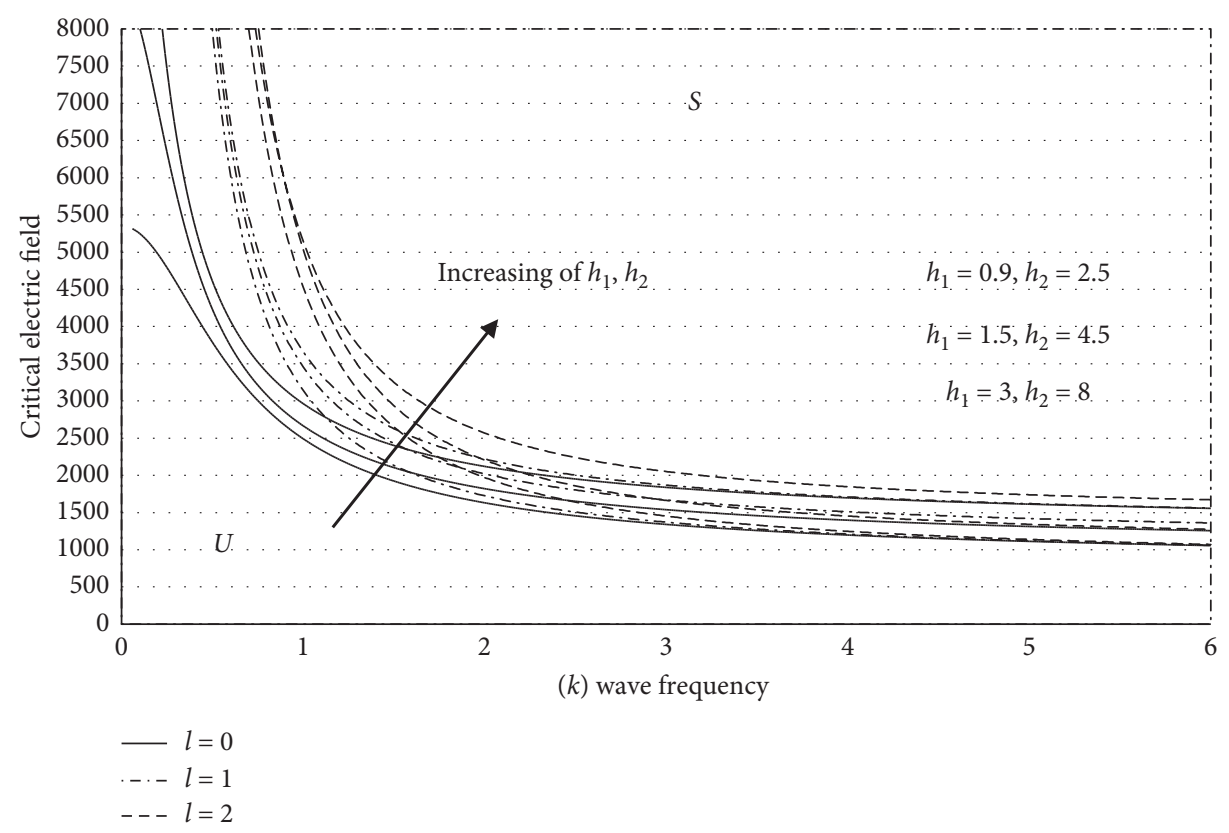

FIgURE 8: Variation of $E_{0}^{2}$ with $k$ for large values of the fluid depths $h_{1}$ and $h_{2}$.

\section{Conclusions}

In this paper, new results of bounded flows through porous medium have been presented and analyzed for weakly electrically conducting fluids. Exact analytical solutions have been given for all parameters.

Using the normal mode analysis, we obtain quadratic dispersion relation of complex coefficients characterizing the behaviour of the disturbed system. Based on appropriate data selections, we conclude the following:

(1) The permeability $\lambda_{1}$ of the medium has no effect on the linear stability of the system

(2) The horizontal electric field $E_{0}^{2}$, porosity of the medium $m$, surface tension $T$, fluid viscosities $\mu_{1}$ and $\mu_{2}$, and electrical conductivites $\alpha_{1}$ and $\alpha_{2}$ have stabilizing effects or enhance the stability

(3) The fluid velocities $U_{1}$ and $U_{2}$ (including the case of absence of fluid velocities $U_{1}=0$ and $U_{2}=0$ or Rayleigh-Taylor instability) have destabilizing effects or tend to reduce the stability

(4) Both the fluid depth $h_{1}$ and $h_{2}$ and the dimension $l$ have a dual role in the system, i.e., stabilizing the system for small values of $h_{1}$ and $h_{2}$, while destabilizing it for large values of $h_{1}$ and $h_{2}$ (including the case of two semiinfinite fluids).

According to the importance of nonlinear effects in EHD phenomena, the describing equations of EHD flow are nonlinear. Therefore, we will discuss the nonlinear stability characteristics for this problem in a subsequent article.

\section{Data Availability}

No data were used to support this study.

\section{Conflicts of Interest}

The authors declare that they have no conflicts of interest.

\section{Acknowledgments}

This project was supported by the Deanship of Scientific Research at Prince Sattam Bin Abdulaziz University under the research project 2017/01/7102.

\section{References}

[1] S. N. Shore, An Introduction to Astrophysical Hydrodynamics, Academic Press Inc., New York, NY, USA, 1992.

[2] S. Chandrasekhar, Hydrodynamic and Hydromagnetic Stability, Oxford University Press, Oxford, UK, 1961.

[3] J. R. Melcher, Field Coupled Surface Waves, MIT Press, Cambridge, MA, USA, 1963.

[4] J. R. Melcher and W. J. Shwarz, "Interfacial relaxation overstability in a tangential electric field," Physics of Fluids, vol. 11, no. 12, pp. 2604-2616, 1968.

[5] J. R. Melcher and G. I. Taylor, "Electrohydrodynamics: a review of the role of Interfacial shear stresses," Annual Review of Fluid Mechanics, vol. 1, no. 1, pp. 111-146, 1969.

[6] J. C. Baygents and F. Baldessari, "Electrohydrodynamic instability in a thin fluid layer with an electrical conductivity gradient," Physics of Fluids, vol. 10, no. 1, p. 301, 1998.

[7] N. Rudraiah and C. O. Ng, "A model for manufacture of nanosized materials free from impurities," Current Science, vol. 86, no. 8, p. 1076, 2004.

[8] J. R. Melcher, Continuum Electromechanics, MIT Press, Cambridge, MA, USA, 1981.

[9] A. A. Mohamed, E. F. Elshehawey, and M. F. El-Sayed, "Electrohydrodynamic stability of two superposed viscous fluids," Journal of Colloid and Interface Science, vol. 169, no. 1, pp. 65-78, 1995. 
[10] G. M. Moatimid, "Stability conditions of an electrified miscible viscous fluid sheet," Journal of Colloid and Interface Science, vol. 259, no. 1, pp. 186-199, 2003.

[11] D. J. Griffiths, Introduction to Electrohydrodynamic, Pearson Education, Delhi, India, 3rd edition, 2006.

[12] M. Jalaal, B. Khorshidi, and E. Esmaeilzadeh, "Electrohydrodynamic (EHD) mixing of two miscible dielectric liquids," Chemical Engineering Journal, vol. 219, pp. 118-123, 2013.

[13] B. M. A. Maher and G. M. Moatimid, "Electro hydro dynamic linear stability of two superposed slightly conducting viscous flows through porous medium with mass and heat transfer," International Journal of Fluid Mechanics, vol. 3, no. 2, pp. 125-134, 2011.

[14] M. F. El-Sayed, A. A. Mohamed, and T. M. N. Metwaly, "Effect of general applied electric field on conducting liquid jets instabilities in the presence of heat and mass transfer," Applied Mathematics and Computation, vol. 172, no. 2, pp. 1078-1102, 2006.

[15] M. F. El-Sayed, A. A. Mohamed, and T. M. N. Metwaly, "Thermohydrodynamic instabilities of conducting liquid jets in the presence of time-dependent transverse electric fields," Physica A: Statistical Mechanics and its Applications, vol. 345, no. 3-4, pp. 367-394, 2005.

[16] L. Fang, X.-Y. Yin, and X.-Z. Yin, "One-dimensional nonlinear instability study of a slightly viscoelastic, perfectly conducting liquid jet under a radial electric field," Physics of Fluids, vol. 28, no. 5, Article ID 053103, 2016.

[17] H. Li, T. N. Wong, and N.-T. Nguyen, "Instability of pressure driven viscous fluid streams in a microchannel under a normal electric field," International Journal of Heat and Mass Transfer, vol. 55, no. 23-24, pp. 6994-7004, 2012.

[18] A. Khan, Z. Shah, S. Islam, S. Khan, W. Khan, and A. Z. Khan, "Darcy-Forchheimer flow of micropolar nanofluid between two plates in the rotating frame with non-uniform heat generation/absorption," Advanced in Mechanical Engineering, vol. 10, no. 10, pp. 1-16, 2018.

[19] F. A. L. Dullien, Fluid Transport and Pore Structure, Academic Press, New York, NY, USA, 1992.

[20] D. B. Ingham and I. Pop, Transport Phenomena in Porous Media, Pergamon Press, Oxford, UK, 1998.

[21] D. A. Nield and A. Bejan, Convection in Porous Media, Springer, Berlin, Germany, second edition, 1999.

[22] K. Vafai, Handbook of Porous Media, Marcel Dekker, New York, NY, USA, 2000.

[23] I. Pop and D. B. Ingham, Convective Heat Transfer: Mathematical and Computational Modeling of Viscous Fluids and Porous Media, Pergamon Press, Oxford, UK, 2000.

[24] R. C. Sharma and T. J. T. Spanos, "The instability of streaming fluids in a porous medium," Canadian Journal of Physics, vol. 60, no. 10, pp. 1391-1395, 1982.

[25] M. F. El-Sayed, "Electrohydrodynamic instability of two superposed viscous streaming fluids through porous media," Canadian Journal of Physics, vol. 75, no. 7, pp. 499-508, 1997.

[26] M. F. El-Sayed, "Effect of normal electric fields on KelvinHelmholtz instability for porous media with Darcian and Forchheimer flows," Physica A: Statistical Mechanics and its Applications, vol. 255, no. 1-2, pp. 1-14, 1998.

[27] M. F. El-Sayed, "Electrohydrodynamic instability of two superposed Walters B' viscoelastic fluids in relative motion through porous medium," Archive of Applied Mechanics (Ingenieur Archiv), vol. 71, no. 11, pp. 717-732, 2001.

[28] M. F. El-Sayed, "Electrohydrodynamic instability of dielectric fluid layer between two semi-infinite identical conducting fluids in porous medium," Physica A: Statistical Mechanics and its Applications, vol. 367, pp. 25-41, 2006.

[29] Y. Gamiel, W. Zahra, and M. El-Behairy, "Stability criteria of streaming conducting fluids through porous media under the influence of a uniform normal magnetic field," International Journal of Mathematics Trends and Technology, vol. 41, no. 2, pp. 147-155, 2017.

[30] M. K. Awasthi, "Three-dimensional magnetohydrodynamic Kelvin-Helmholtz instability of cylindrical flow with permeable boundaries," Physics of Plasmas, vol. 21, Article ID 032124, 2014.

[31] M. F. El-Sayed, G. M. Moatimid, and T. M. N. Metwaly, "Nonlinear Kelvin-Helmholtz instability of two superposed dielectric finite fluids in porous medium under vertical electric fields," Chemical Engineering Communications, vol. 197, no. 5, pp. 656-683, 2010.

[32] M. F. El-Sayed, G. M. Moatimid, and T. M. N. Metwaly, "Nonlinear electrohydrodynamic stability of two superposed streaming finite dielectric fluids in porous medium with interfacial surface charges," Transport in Porous Media, vol. 86, no. 2, pp. 559-578, 2011.

[33] M. F. El-Sayed, N. T. Eldabe, M. H. Haroun, and D. M. Mostafa, "Nonlinear electroviscoelastic potential flow instability theory of two superposed streaming dielectric fluids," Canadian Journal of Physics, vol. 92, no. 10, pp. 1249-1257, 2014.

[34] K. Zakaria, M. A. Sirwah, and S. Alkharashi, "Instability through porous media of three layers superposed conducting fluids," European Journal of Mechanics-B/Fluids, vol. 28, no. 2, pp. 259-270, 2009.

[35] Y. Abd Elmaboud, "Two layers of immiscible fluids in a vertical semi-corrugated channel with heat transfer: impact of nanoparticles," Results in Physics, vol. 9, pp. 1643-1655, 2018.

[36] Y. Abd Elmaboud, S. I. Abdelsalam, K. S. Mekheimer, and K. Vafai, "Electromagnetic flow for two-layer immiscible fluids," Engineering Science and Technology, an International Journal, vol. 22, no. 1, pp. 237-248, 2019.

[37] Z. Zahreddine and E. F. El-Shehawey, "On the stability of a system of differential equations with complex coefficients," Indian Journal of Pure and Applied Mathematics, vol. 19, pp. 963-972, 1988.

[38] G. M. Moatimid and M. H. Obied Allah, "Electrohydrodynamic linear stability of finitely conducting flows through porous fluids with mass and heat transfer," Applied Mathematical Modelling, vol. 34, no. 10, pp. 3118-3129, 2010. 